\title{
Postioplastia circunferencial para correção de fimose congênita em gato: Relato de
}

\section{Caso}

\author{
Circumferential postioplasty for correction of congenital phimosis in cats: Case Report \\ Postioplastia circunferencial para la corrección de la fimosis congénita en gatos: Reporte de caso
}

Recebido: 11/01/2021 | Revisado: 13/01/2021 | Aceito: 17/01/2021 | Publicado: 21/01/2021

Maíra Planzo Fernandes

ORCID: https://orcid.org/0000-0002-2449-6990

Universidade Estadual de Londrina, Brasil

E-mail: maira.fernandes.planzo@uel.br

Maria Isabel Mello Martins

ORCID: https://orcid.org/0000-0001-8416-2450

Universidade Estadual de Londrina, Brasil

E-mail: imartins@uel.br

Julia Rodrigues Greghi

ORCID: https://orcid.org/0000-0002-9596-2426

Universidade Estadual de Londrina, Brasil

E-mail: julia.rodrigues.greghi@uel.br

Aline Groth

ORCID: https://orcid.org/0000-0002-3177-6851

Universidade Estadual de Londrina, Brasil E-mail: aline.groth100@uel.br

Guilherme Schiess Cardoso

ORCID: https://orcid.org/0000-0002-6096-1642

Universidade Estadual de Londrina, Brasil E-mail: schiess@uel.br

Camila da Costa Gomes

ORCID: https://orcid.org/0000-0002-6286-6411 Universidade Estadual de Londrina, Brasil

E-mail: camila.costa.gomes@uel.br

Vinícius Wagner Silva

ORCID: https://orcid.org/0000-0001-8623-5131 Universidade Estadual de Londrina, Brasil E-mail: viniciuswagner.vet@gmail.com

Luana Martins de Souza Amaral

ORCID: https://orcid.org/0000-0001-9691-3450

Universidade Estadual de Londrina, Brasil

E-mail: luanamsa20@gmail.com

Natalia Ribeiro da Silva

ORCID: https://orcid.org/0000-0002-5117-5906

Universidade Estadual de Londrina, Brasil

E-mail: natalia.ribeiro@uel.br

\begin{abstract}
Resumo
O objetivo do trabalho foi relatar a apresentação clínica e, a correção cirúrgica da fimose congênita em um gato jovem, por postioplastia circunferencial. Um gato, macho, sem raça definida, com quatro meses de vida, deu entrada no Hospital Veterinário da Universidade Estadual de Londrina, com queixa de disúria, polaciúria e polidipsia desde o nascimento, além de hematúria há um dia. No exame físico foi observada dermatite úmida em região escrotal, com presença de urina na cavidade prepucial, e, ao tentar-se a tração do pênis do paciente para a avaliação, foi identificado orifício prepucial com abertura insuficiente para a exposição. Diante do histórico, da idade do paciente e exame físico, fimose por estenose congênita do óstio prepucial foi sugerida como diagnóstico. $O$ tratamento instituído foi a cirurgia de correção. Realizada uma incisão em forma de cunha na face craniodorsal do prepúcio com exérese de cerca de $3 \mathrm{~mm}$, a mucosa da borda ipsilateral do prepúcio foi posicionada em padrão de sutura simples descontínua, utilizando fio não absorvível (monoylon) 5-0. Dez dias depois do procedimento, o animal estava urinando em jato, sem mais alterações. Desta forma, a realização da correção cirúrgica de fimose congênita em gatos, com a técnica de postioplastia circunferencial, é recomendada, pois há resolução das alterações clínicas. Além disso, foi evidente no presente trabalho, que o sucesso do prognóstico do paciente se deu devido ao conhecimento da fisiologia e a um exame clínico pediátrico eficiente.
\end{abstract}

Palavras-chave: Disúria; Felino; Jovem; Pênis; Prepúcio. 


\begin{abstract}
The objective of the study was to report the clinical presentation and, the surgical correction of congenital phimosis in a young cat, by circumferential postioplasty. A cat, male, mixed breed, with four months of life, was admitted to the Veterinary Hospital of the State University of Londrina, complaining of dysuria, polyuria and polydipsia since birth, in addition to hematuria one day ago. On physical examination, wet dermatitis was observed in the scrotal region, with the presence of urine in the preputial cavity, and when trying to pull the patient's penis for the evaluation, a preputial orifice with insufficient opening for exposure was identified. In view of the history, the patient's age and physical examination, phimosis due to congenital stenosis of the preputial ostium was suggested as a diagnosis. The treatment instituted was correction surgery. A wedge-shaped incision was made in the craniodorsal face of the foreskin with an excision of about $3 \mathrm{~mm}$, the mucosa of the ipsilateral edge of the foreskin was positioned in a simple discontinuous suture pattern, using a 5-0 non-absorbable suture. Ten days after the procedure, the animal was urinating in a jet, without further changes. Thus, performing the surgical correction of congenital phimosis in cats, using the circumferential postioplasty technique, is recommended, as there is resolution of the clinical changes. In addition, it was evident in the present study that the patient's prognosis success was due to the knowledge of physiology and an efficient pediatric clinical examination.
\end{abstract}

Keywords: Dysuria; Feline; Young; Penis; Foreskin.

\title{
Resumen
}

El objetivo del estudio fue reportar la presentación clínica y la corrección quirúrgica de la fimosis congénita en un gato joven, mediante posioplastia circunferencial. Un gato, macho, mestizo, con cuatro meses de vida, fue ingresado en el Hospital Veterinario de la Universidad Estatal de Londrina, quejándose de disuria, poliuria y polidipsia desde su nacimiento, además de hematuria hace un día. Al examen físico se observó dermatitis húmeda en la región escrotal, con presencia de orina en la cavidad prepucial, y al intentar tirar del pene del paciente para la evaluación se identificó un orificio prepucial con apertura insuficiente para la exposición. Teniendo en cuenta la historia, la edad y la exploración física del paciente, se sugirió como diagnóstico la fimosis por estenosis congénita del ostium prepucial. El tratamiento instituido fue la cirugía correctora. Se realizó una incisión en forma de cuña en la cara craneodorsal del prepucio con escisión de unos $3 \mathrm{~mm}$, se colocó la mucosa del borde ipsilateral del prepucio en un patrón de sutura discontinua simple, utilizando una sutura no absorbible 5-0. Diez días después del procedimiento, el animal estaba orinando a chorro, sin mayores cambios. Así, se recomienda realizar la corrección quirúrgica de la fimosis congénita en gatos, mediante la técnica de posioplastia circunferencial, ya que existe resolución de los cambios clínicos. Además, fue evidente en el presente estudio que el éxito del pronóstico del paciente se debió al conocimiento de la fisiología y un examen clínico pediátrico eficiente.

Palabras clave: Disuria; Felino; Joven; Pene; Prepúcio.

\section{Introdução}

A fimose não é uma condição comum em cães e gatos e é definida como a inabilidade de exposição do pênis a partir da bainha prepucial, comumente resultante de um orifício prepucial estreito, podendo ser de forma congênita ou adquirida (Bojrab, 2014). Acredita- se que a fimose adquirida é resultante de trauma, como, por exemplo excesso de higiene, ou por sequela de neoplasia e celulite (May \& Hauptman, 2009).

A fimose congênita pode ser resultante de uma anormalidade do desenvolvimento do pênis ou prepúcio, como presença de um pênis curto, aderências entre o prepúcio e o pênis e, estenose ou ausência do orifício prepucial (Arthur, Noakes, \& Pearson, 1996).

Os sinais clínicos desse tipo de fimose incluem retenção urinária, levando a distensão do prepúcio e, consequente balonopostite, além de dificuldade em copular, devido à falta de capacidade em exteriorizar o pênis (De La Puerta \& Baines, 2012). O diagnóstico é realizado com base nos achados da anamnese e exame físico (Robson, 2005), e os diferenciais são a hipoplasia peniana e o hermafroditismo (Weide, Contesini, Ferreira, \& Stedile, 2006).

O tratamento de fimose é cirúrgico, com indicação da técnica de postioplastia circunferencial, caso não haja aderência entre pênis e prepúcio, e a postioplastia, seguida de urestrostomia prepucial, caso exista aderência entre prepúcio e pênis (De Vlaming, Wallace \& Ellison, 2019).

As doenças congênitas estão entre as principais causas de morte neonatal de pequenos animais (Souza, 2017), tornando imprescindível uma maior atenção dos médicos veterinários nessa fase da vida. Ao conhecimento dos autores existem poucos trabalhos que relataram a ocorrência de fimose em filhotes de gatos (Bastos et al., 2020; Bright \& Mellanby, 2004; De Vlaming 
et al., 2019; May \& Hauptman, 2009; Yoon \& Jong, 2013).

Diante da necessidade em aproximar o clínico veterinário com as enfermidades neonatais, objetivou-se, no presente trabalho, descrever a apresentação clínica e, a correção cirúrgica efetiva da fimose congênita em um gato jovem, através da postioplastia circunferencial.

\section{Metodologia}

Este trabalho trata-se de um relato de caso de caráter descritivo e natureza qualitativa, já que a pesquisa ocorreu em ambiente natural, com coleta direta de dados descritivos e o pesquisador foi o principal instrumento (Ludke \& Andre, 2013). Os dados foram coletados através dos registros e exames fornecidos pelo atendimento médico veterinário no Hospital Veterinário da Universidade Estadual de Londrina.

\section{Relato de Caso}

Um gato, macho, sem raça definida, com quatro meses de vida e pesando $1,3 \mathrm{~kg}$, foi atendido devido a disúria, polaciúria e polidipsia desde o nascimento, além de hematúria há um dia. O proprietário relatou que havia adotado o animal há 1 mês e que os irmãos da ninhada apresentavam má formações no pênis. Durante exame físico foi notado algumas alterações, como: dermatite úmida em região escrotal, contendo urina na cavidade prepucial, no qual estão ilustradas na Figura 1. No presente momento tentou-se expor o pênis do paciente, porém foi identificado orifício prepucial com abertura insuficiente (cerca de 0,8 milímetros de diâmetro), não sendo possível realizar a exposição.

Figura 1 - Fotografia do prepúcio do gato com fimose congênita. Observe a presença da abertura mínima do orifício prepucial (seta vermelha).

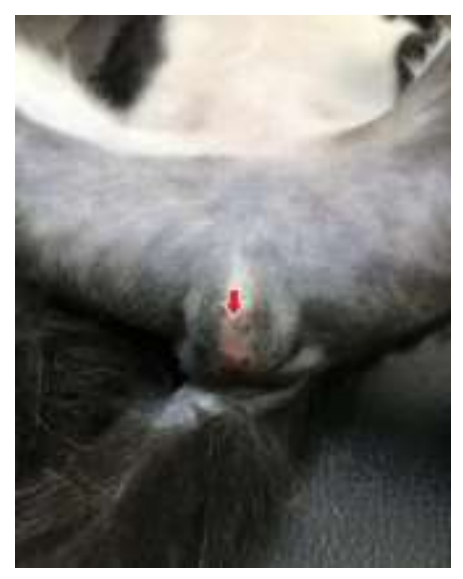

Fonte: Autores.

Após exame físico, foi colhido por venopunção da jugular amostra sanguínea para hemograma e exames bioquímicos, ureia e creatinina, tais exames se encontravam dentro da normalidade para a espécie. Foi realizada ultrassonografia abdominal, no qual foi visibilizado pequena quantidade de sedimento em bexiga, com parede lisa e regular. Demais órgãos sem alterações. A urina foi colhida por cistocentese, e enviada para urinálise. O exame revelou traços de proteína, bacteriúria (++) e 40 leucócitos por campo. Devido as alterações encontradas em urina e bexiga, foi instituído antibioticoterapia com Amoxicilina + Ácido Clavulânico (12,5 mg/kg, a cada 12 horas, por 10 dias). Depois de sete dias do início do tratamento, foi colhida nova amostra de urina e enviada para cultura, cujo resultado foi negativo para crescimento bacteriano.

Com base no histórico da ninhada, a idade do paciente e, com base na anamnese e exame físico, fimose por estenose 
congênita do óstio prepucial foi sugerida como diagnóstico.

O tratamento instituído foi a cirurgia de correção. Como medicação pré-anestésica foi administrado $0,2 \mathrm{mg} / \mathrm{kg}$ de sulfato de morfina (Dimorf®), intramuscular, seguido de indução anestésica com propofol (Lipuro 1\%®), 10mg/kg, por via intravascular. Após isso, o paciente foi intubado, com tubo endotraqueal $\mathrm{n}^{\circ} 3$, e realizado bloqueio epidural com $0,15 \mathrm{mg} / \mathrm{kg}$ de cloridato de bupivacaína (Neocaína®) e $0,1 \mathrm{mg} / \mathrm{kg}$ de sulfato de morfina (Dimorf®). A manutenção foi feita com isofluorano (Isoforine ${ }^{\circledR}$ ) a oxigênio $100 \%$, no sistema avalvular. Foi administrado $30 \mathrm{mg} / \mathrm{kg}$ cefazolina sódica (Kefazol®), por via intravenosa, como antibioticoterapia pré-operatória. Durante todo o procedimento o animal foi mantido na fluidoterapia com Ringer Lactato na taxa de $5 \mathrm{ml} / \mathrm{kg} / \mathrm{h}$.

Com o paciente em decúbito dorsal foi realizada a preparação da área cirúrgica com tricotomia das regiões inguinal, do prepúcio e perianal. Em seguida foi feita antissepsia com clorexidine degermante (Riohex 2\%®) e posteriormente, com Álcool a 70\% e clorexidine alcoólico (Riohex 0,5\%®). Em relação a técnica cirúrgica foi realizada uma incisão em forma de cunha na face craniodorsal do prepúcio com exérese de cerca de $3 \mathrm{~mm}$. A mucosa foi divulsionada, com uma tesoura Metzenbaum, até o pênis ser acessado. A Figura 2 corresponde a esse momento do período transoperatório.

Figura 2 - Fotografia da incisão em forma de cunha em prepúcio e retirada do seu fragmento, durante o transoperatório da correção cirúrgica de fimose congênita em gato.

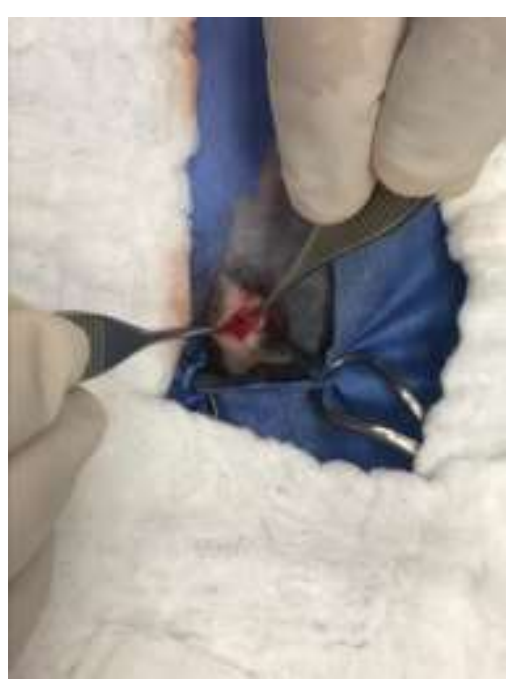

Fonte: Autores.

O pênis foi inspecionado e não foram encontradas quaisquer alterações, nem aderências. Após isso, a mucosa da borda ipsilateral do prepúcio foi posicionada em padrão de sutura simples descontínua, utilizando fio não absorvível (monoylon) 5-0 (situação essa caracterizada na Figura 3). 
Figura 3 - Fotografia do padrão de sutura simples descontínua, com fio não absorvível, em borda de mucosa do prepúcio do gato acometido por fimose congênita.

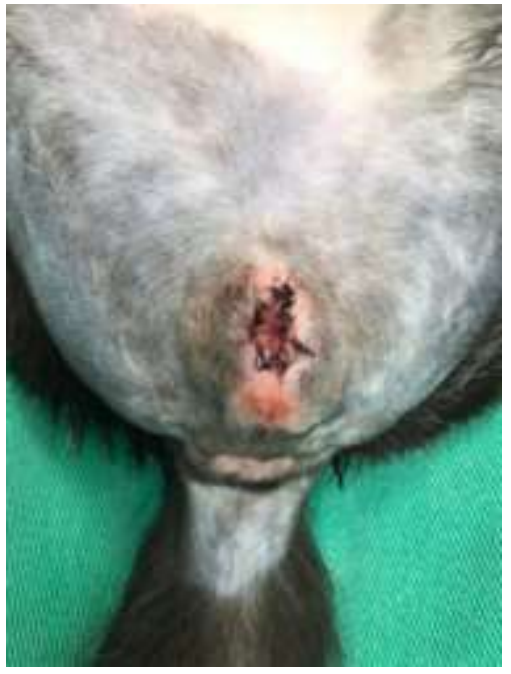

Fonte: Autores.

Como medicação pós-operatória foi realizada $0,2 \mathrm{mg} / \mathrm{kg}$ de meloxicam (Eloxicam 0,2\%®), 0,2 mg/kg de cloridato de metadona (Mytedom $\left.{ }^{\circledR}\right)$ e $25 \mathrm{mg} / \mathrm{kg}$ de dipirona sódica (Febrax $\left.{ }^{\circledR}\right)$, todas por via subcutânea. Paciente ficou internado por 24 horas para observação da micção e para analgesia com cloridrato de tramadol (Tramal@) a cada 8 horas, e dipirona a cada 12 horas, ambos por via intravenosa.

Após as 24 horas, animal estava urinando em jato e sem hematúria. Desta forma, paciente teve alta médica, sendo prescrito dipirona gotas (1 gota/kg, a cada 12 horas, por 5 dias), por via oral, limpeza dos pontos diariamente com solução fisiológica seguida de aplicação de digliconato de clorexidina 1\% (Methiolate®), além do uso de colar Elizabetano. Três dias após a alta foi marcado retorno para avaliação clínica e inspeção da sutura. Proprietário relatou que animal estava urinando em jato, na caixa sanitária, e não havia alterações dignas de nota. Dez dias depois do procedimento, a incisão estava em bom estado de cicatrização, propiciando a retirada dos pontos.

\section{Discussão}

A fimose é uma alteração mais comum em pacientes humanos do que em cães, e em felinos é ainda mais improvável (Volpato, Ramos, Magalhães, Lopes, \& Souza, 2010). Quando acontecem nessa espécie, geralmente são de natureza congênita, sendo necessária correção cirúrgica para evitar problemas futuros, principalmente balanopostite (Weide et al., 2006).

Frequentemente a fimose congênita ocorre em animais pediátricos, porém tal fato não é confirmatório para o seu diagnóstico, pois a lambedura excessiva da mãe e dos irmãos pode causar trauma em prepúcio (May \& Hauptman, 2009). No presente estudo, embora o animal fosse jovem, quatro meses de idade, o proprietário relatou que a ninhada também apresentava outras más formações, reforçando o diagnóstico de fimose congênita e, não de fimose adquirida.

A fimose congênita pode ser resultante de uma anormalidade do desenvolvimento do pênis ou prepúcio. Essas anomalias incluem a presença de um pênis curto, aderências entre o prepúcio e o pênis e, estenose ou ausência do orifício prepucial (Arthur et al., 1996). No presente relato, a fimose congênita foi decorrente de uma estenose do orifício, assim como descrito em trabalho prévios (Bright \& Mellanby, 2004; De Vlaming et al., 2019; May \& Hauptman, 2009; Yoon \& Jong, 2013). Além disso, o animal do presente estudo apresentava em seu exame clínico polaciúria, prepúcio eritematoso, devido a dermatite úmida causada pela acumulação de urina, e incapacidade de expor o pênis (características também observadas nos estudos citados anteriormente). 
Em um relato, descrevendo fimose congênita em um gato de dois meses, foi encontrado Escherichia coli na cultura urinária, além de hematúria em urinálise (Bright \& Mellanby, 2004). No mesmo trabalho, devido aos achados instituiu-se antibioticoterapia com Amoxicilina, $10 \mathrm{mg} / \mathrm{kg}$, a cada 12 horas. Setenta e cinco dias após o início do tratamento foi repetido a cultura, tendo resultado negativo para crescimento bacteriano. Os autores acreditam que a compressão do pênis no interior do prepúcio provavelmente causava acúmulo de urina e, predispôs o gato a desenvolver uma infecção ascendente da bexiga. O mesmo fato pode ser explicado no presente trabalho. A urinálise e ultrassonografia abdominal, no primeiro atendimento, revelaram traços de proteína, sangue e de bactérias na urina, coletada por cistocentese, além de presença de sedimento em bexiga. Já que os sinais eram compatíveis com cistite, foi realizado antibioticoterapia e, sete dias depois do tratamento, o resultado da urinálise foi negativo na cultura.

Após o emprego da antibioticoterapia, a hematúria e polaciúria se tornaram ausentes no paciente, no qual pode-se sugerir que tais achados são resultantes de uma infecção no trato urinário inferior, associada a fimose.

Para correção da fimose, realizou-se a técnica de postioplastia circunferencial, assim como De Vlaming et al., 2019.

No presente estudo, o paciente foi acompanhado 20 dias depois da cirurgia e apresentava exposição da glande do pênis, conforme mostra a Figura 4.

Figura 4 - Fotografia da exposição de ponta de pênis vinte dias após cirurgia para correção de fimose congênita em gato.

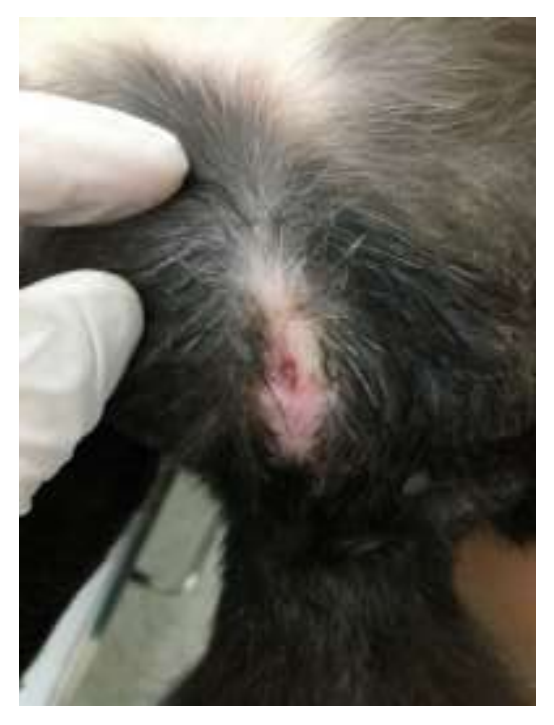

Fonte: Autores.

Tal alteração também pôde ser vista em outro relato, dez dias após a cirurgia (Bright \& Mellanby, 2004). Segundo De Vlaming et al., 2019, dos seis gatos acometidos por tal complicação, cinco deles tiveram a glande do pênis recoberta pelo prepúcio após 25 dias de pós-operatório, não sendo, portanto, uma alteração relevante.

No presente estudo em três meses de pós-operatório o tutor do paciente não informou qualquer alteração na micção do animal, divergindo das alterações previamente descritas, de infecção do trato urinário três semanas após (May \& Hauptman, 2009), e disúria sete meses após a cirurgia de correção da fimose (De Vlaming et al., 2019). Os dois trabalhos mencionados não conseguiram encontrar associação para o surgimento de disúria/infecção do trato urinário no pós-operatório dos pacientes.

Até o momento, em todos os relatos sobre correção de fimose em gatos, utilizou-se fio absorvível para suturar a mucosa da borda ipsilateral do prepúcio (Bright \& Mellanby, 2004; De Vlaming et al., 2019; May \& Hauptman, 2009; Yoon \& Jong, 2013). O presente estudo, diferentemente, utilizou fio não absorvível para realização dessa técnica. Convergindo com o nosso estudo, dois trabalhos utilizaram o mesmo tipo de fio, porém para correção de fimose em cães (Weide et al., 2006; Hungria, 
2013).

Não foi realizada a orquiectomia do paciente no momento da correção cirúrgica, com o objetivo de aguardar a maturidade sexual, não interferindo, portanto, no desenvolvimento dos hormônios sexuais do animal.

\section{Conclusão}

Desta forma, a realização da correção cirúrgica de fimose congênita em gatos, utilizando a técnica de postioplastia circunferencial, é recomendada, pois há resolução das alterações clínicas. A complicação pós-operatória é mínima e de fácil resolução.

O paciente consegue urinar sem dificuldade logo após o procedimento, diminuído assim a probabilidade de infecção local, dando-lhe qualidade de vida.

Além disso, foi evidente no presente trabalho, que o sucesso do prognóstico do paciente se deu devido ao conhecimento da fisiologia e a um exame clínico pediátrico eficiente.

Novos trabalhos precisam ser relatados a fim de levar conhecimento, sobre outras complicações pós-operatória, aos médicos veterinários e, com isso, diminuir as falhas do tratamento médico.

\section{Referências}

Arthur, G. H., Noakes, D. E., Pearson, H., \& Parkinson, T. J. (1996). Veterinary Reproduction and Obstetrics. (7a ed.): Saunders, $714-24$.

Bojrab, M. J. (2014). Mecanismos das doenças em cirurgia de pequenos animais. (3a ed.): Roca, 1040p.

Bastos, M. M. S., Pantoja, A. R., Everton, E. B., Carneiro, M. J. C., \& Aires, E. O. M. (2020). Postioplastia por circuncisão para redução de fimose em gato: relato de caso. Medicina Veterinária (UFRPE), 14(2), 113-116.

Bright, S. R., \& Mellanby, R. J. (2004). Congenital phimosis in a cat. Journal of feline medicine and surgery, 6(6), 367-370.

De Vlaming, A., Wallace, M. L., \& Ellison, G. W. (2019). Clinical characteristics, classification, and surgical outcome for kittens with phimosis: 8 cases (20092017). Journal of the American Veterinary Medical Association, 255(9), 1039-1046.

May, L. R., \& Hauptman, J. G. Phimosis in cats: 10 cases (2000- 2008). J Am Anim Hosp Assoc 2009; 45:277-283.

Freitas, P. M. C., Luz, M. R., Paraguassú, A. O., \& Barbosa, B. C. (2019). Particularidades nas cirurgias do sistema reprodutor da espécie canina. Rev. Bras. Reprod. Anim, 43(2), 346-355.

Fossum, T. W. (2002). Small Animal Surgery. (2nd ed), Mosby, 11830 Westline Industrial Drive, St. Louis, Missouri-63146, 297.

De la Puerta, B., \& Baines, S. (2012). Surgical diseases of the genital tract in male dogs 2. Penis and prepuce. In Practice, 34(3), $128-135$.

De Souza, T. D. (2017). Mortalidade fetal e neonatal canina.

Hobson, H. P. Penis and prepuce. In: Bojrab M.J., editor. Current techniques in small animal surgery. Baltimore: Williams and Wilkins; $2005,527-37$.

Hungria, C. B. Postioplastia com punch de biópsia para a correção cirúrgica de fimose causada por estenose congênita do óstio prepucial em um cão: relato de caso.

Lundke, M., \& Andre, M. E. D. A.(2013). Pesquisa em educação: uma abordagem qualitativa. (2a ed.): EPU

Weide, L. A., Contesini, E. A., Ferreira, M. P., \& Stedile, R. (2006). Postioplastia modificada para a redução de fimose em cães. Acta Scientiae Veterinariae, 34(3), 339-342.

Volpato, R., Ramos, R. D. S., Magalhães, L. C. O., Lopes, M. D., \& Souza, D. B. D. (2010). Afecções do pênis e prepúcio dos cães: revisão de literatura. Veterinária e Zootecnia, 312-323.

Yoon, H. Y., \& Jeong, S. W. (2013). Surgical correction of a congenital or acquired phimosis in two cats. 한국임상수의학회지, 30(2), 123-126. 